\title{
ESO VLT optical spectroscopy of BL Lac objects
}

\section{Boris Sbarufatti}

INAF, Istituto di Astrofisica Spaziale e Fisica Cosmica di Palermo, Via Ugo La Malfa 153,

I-90146 Palermo, Italy

E-mail: sbarufatti@ifc.inaf.it

\section{Stefano Ciprini}

1. Physics Department University of Perugia, and I.N.F.N. Perugia Section, Via A. Pascoli, I-06123 Perugia, Italy

2. Tuorla Observatory, University of Turku, Väisäläntie 20, FIN-21500 Piikkiö,Finland

E-mail: stefano.ciprini@pg.infn.it

\section{Jari Kotilainen}

Tuorla Observatory, University of Turku, Väisäläntie 20, FIN-21500 Piikkiö,Finland

E-mail: jarkot@utu.fi

\section{Roberto Decarli}

Università degli Studi dell'Insubria, via Valleggio 11, I-22100 Como, Italy

E-mail: roberto.decarli@mib.infn.it

\section{Aldo Treves*}

Università degli Studi dell'Insubria, via Valleggio 11, I-22100 Como, Italy

E-mail: aldo.treves@uninsubria.it

\section{Angelo Veronesi}

Università degli Studi dell'Insubria, via Valleggio 11, I-22100 Como, Italy

E-mail: averonesi@dfm. uninsubria.it

\section{Renato Falomo}

INAF, Osservatorio Astronomico di Padova, Vicolo dell'Osservatorio 5, I-35122 Padova, Italy

E-mail: renato.falomo@oapd.inaf.it

Using ESO VLT plus FORS 1 for spectroscopy of BL Lac objects of unknown $z$, we measured the redshift of 4 targets and lower limits for 8 others.

Workshop on Blazar Variability across the Electromagnetic Spectrum April 22-25 2008

Palaiseau, France

\footnotetext{
*Speaker.
} 


\section{Observations, analysis and results}

We present results of an ongoing program at the ESO VLT plus FORS 1 for high S/N spectroscopy of BL Lac objects lacking of a firm redshift estimate, performed in non optimal seeing conditions (see Sbarufatti et al. 2005 ${ }^{[1]}, 2006^{[2]}$ ).

We report on 12 new sources for which we confirm the BLL classification. New redshifts are determined for 4 objects, 2 with weak emission lines (PKS 1057-79, $z=0.569$; TXS 2346+052, $z=0.419$ ) and 2 with absorptions from the host galaxy (RBS 1752, $z=0.449$; RBS 1915, $z=$ 0.243), see Table 1 and Figure 1.

For the remaining 8 BL Lacs, from the very absence of absorption lines of the host galaxy, lower limits to the redshift are deduced with $z_{\min }$ in the interval $0.20-0.80$, see Sbarufatti et al. $2006^{[2]}$ and Table 2.

A detailed description of the adopted techniques and results is contained in Sbarufatti et al. $2008^{[3]}$. All the spectra of our program can be retrieved at http: / www. oapd. inaf. it/zbllac/.

\begin{tabular}{ll}
\hline \hline Object name & redshift \\
\hline PKS 1057-79 & $z=0.569$ \\
RBS 1752 & $z=0.449$ \\
RBS 1915 & $z=0.243$ \\
TXS 2346+052 & $z=0.419$ \\
\hline
\end{tabular}

Table 1: Redshifts for BL Lac objects

\begin{tabular}{ll}
\hline \hline Object name & $\begin{array}{l}\text { lower limits } \\
\text { to the redshift }\end{array}$ \\
\hline PKS 0019+058 & $z>0.4$ \\
GC 0109+224 & $z>0.2$ \\
RBS 0231 & $z>0.4$ \\
OM 280 & $z>0.2$ \\
OQ 012 & $z>0.5$ \\
PMNJ 1539-0658 & $z>0.8$ \\
PKS 1830-589 & $z>0.5$ \\
1RXS J235730.1-171801 & $z>0.6$ \\
\hline
\end{tabular}

Table 2: Lower limits to the redshifts for BL Lac objects without spectral features 


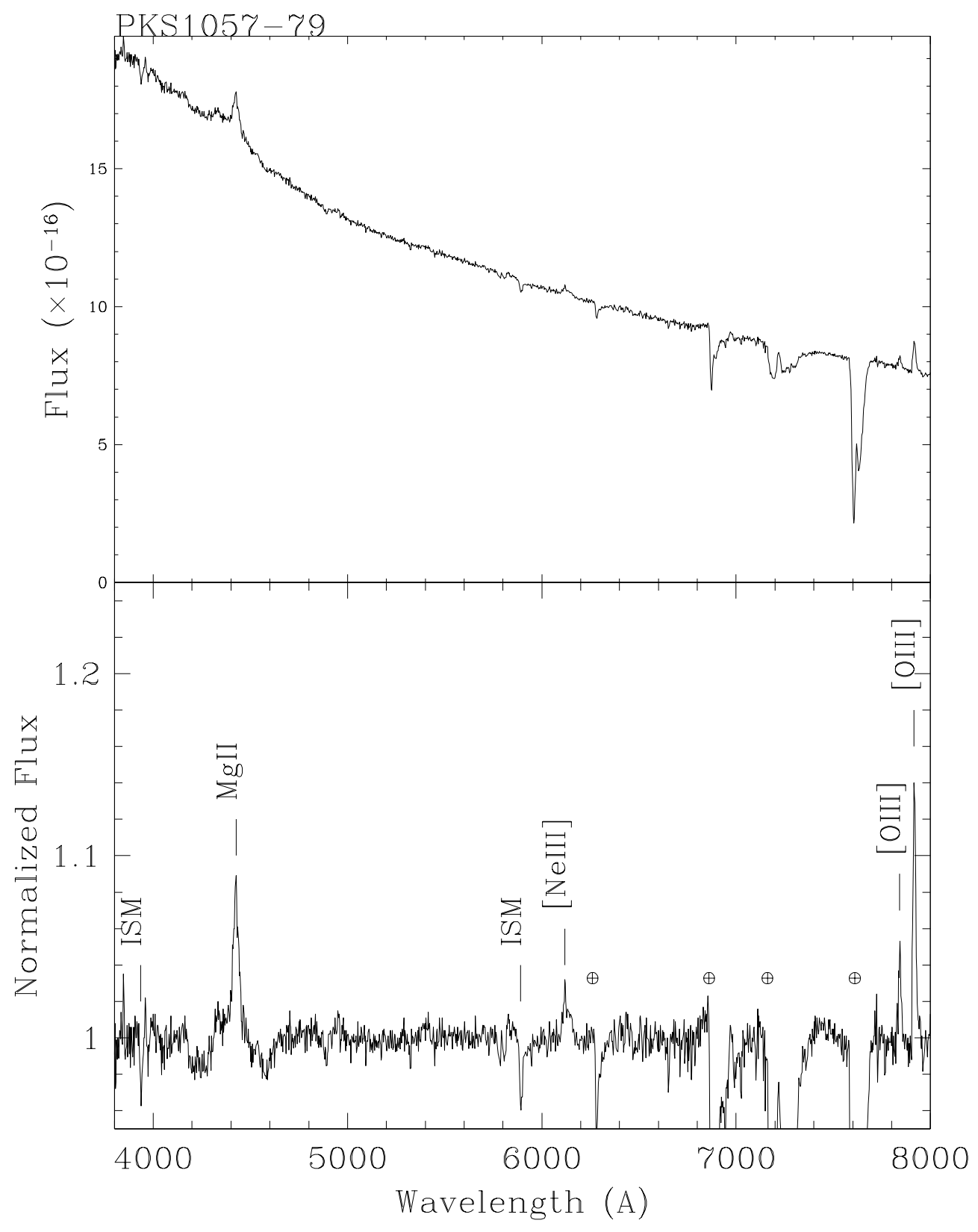

Figure 1: Top panels: flux calibrated dereddened spectra; the flux is measured in units of $\mathrm{erg} / \mathrm{cm}^{2} / \mathrm{s} / \AA$. Bottom panels: normalized spectra. Telluric bands are indicated by $\oplus$, spectral features are marked by the line identifi cations. 


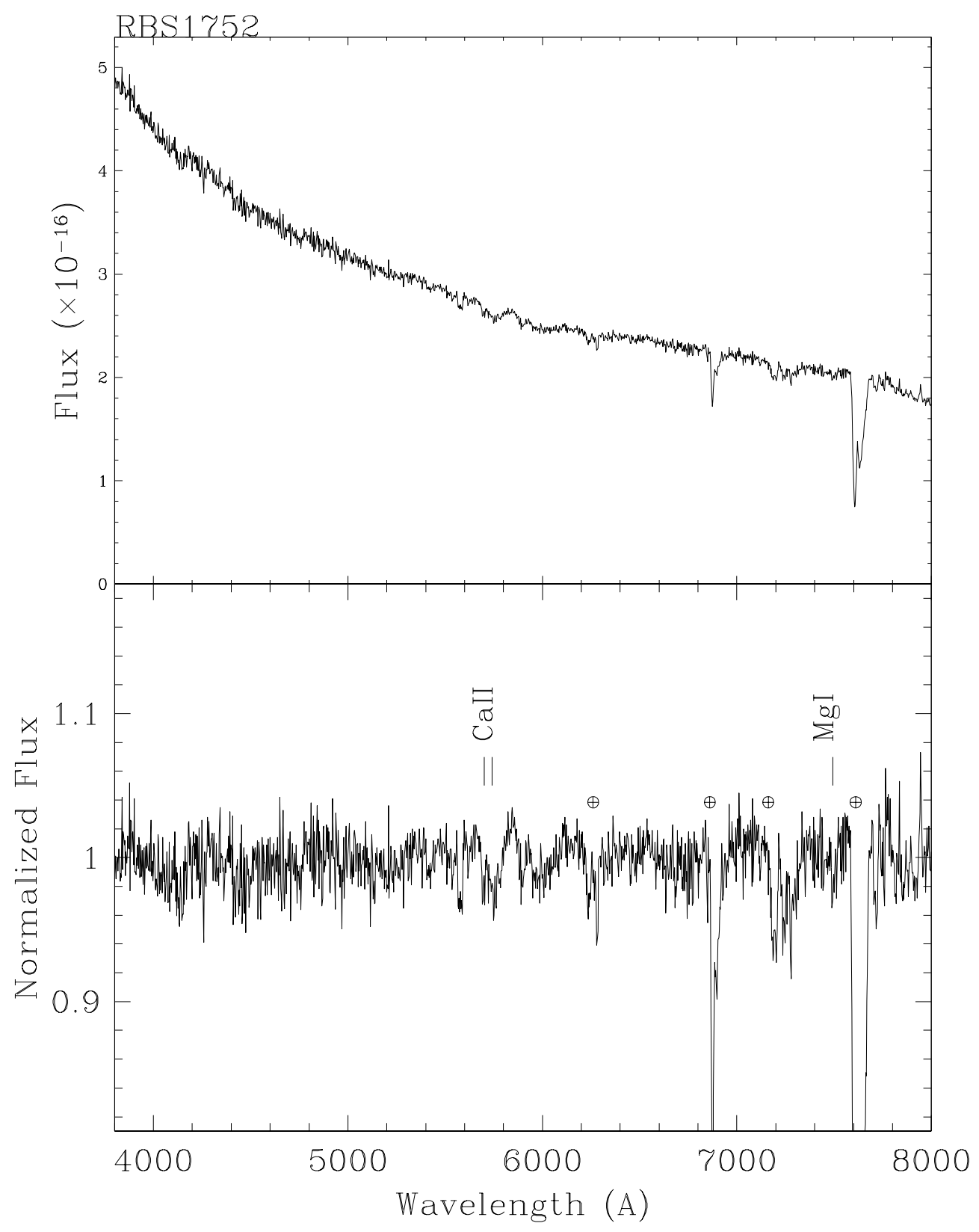

Figure 1:-continued. 


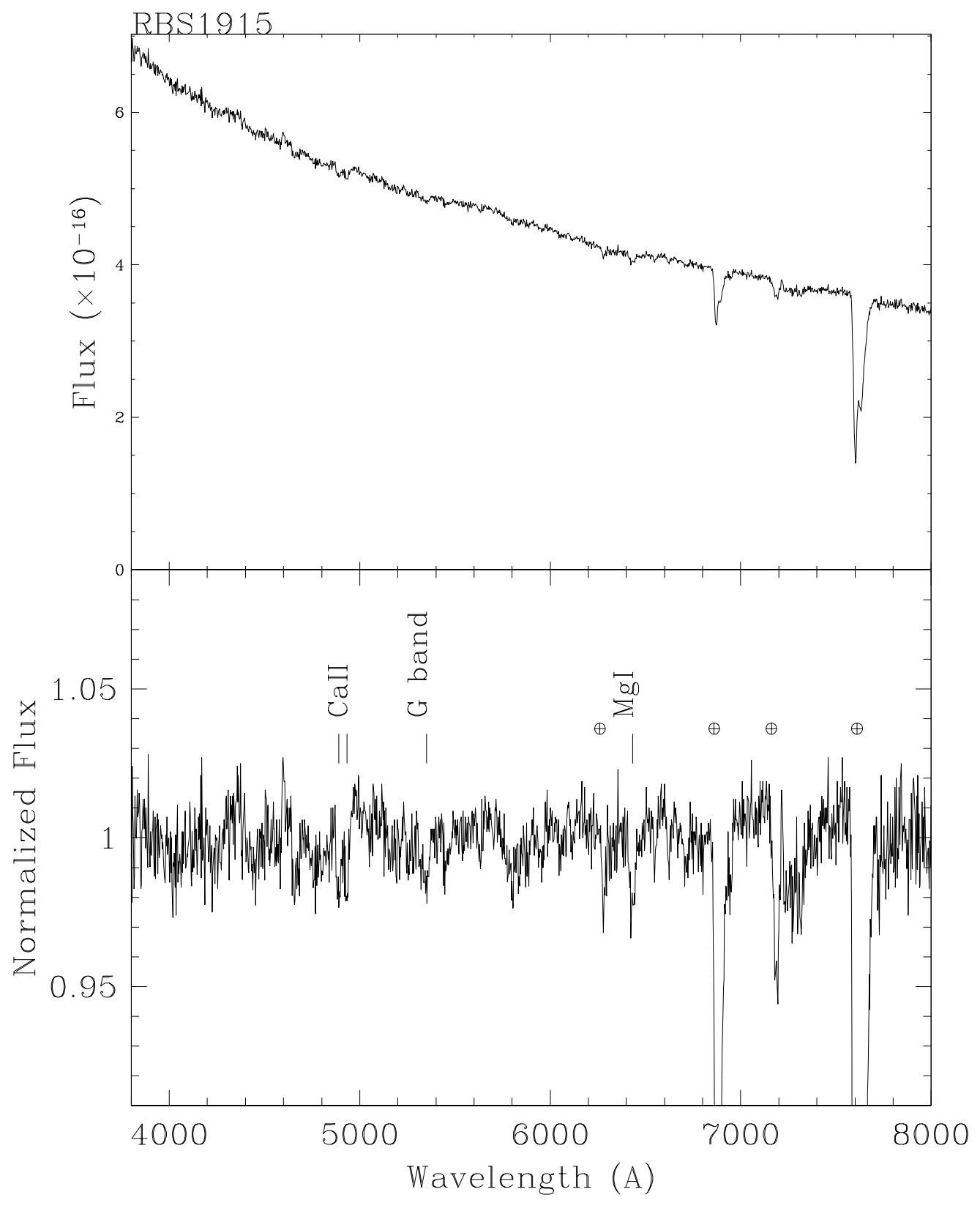

Figure 1:-continued. 


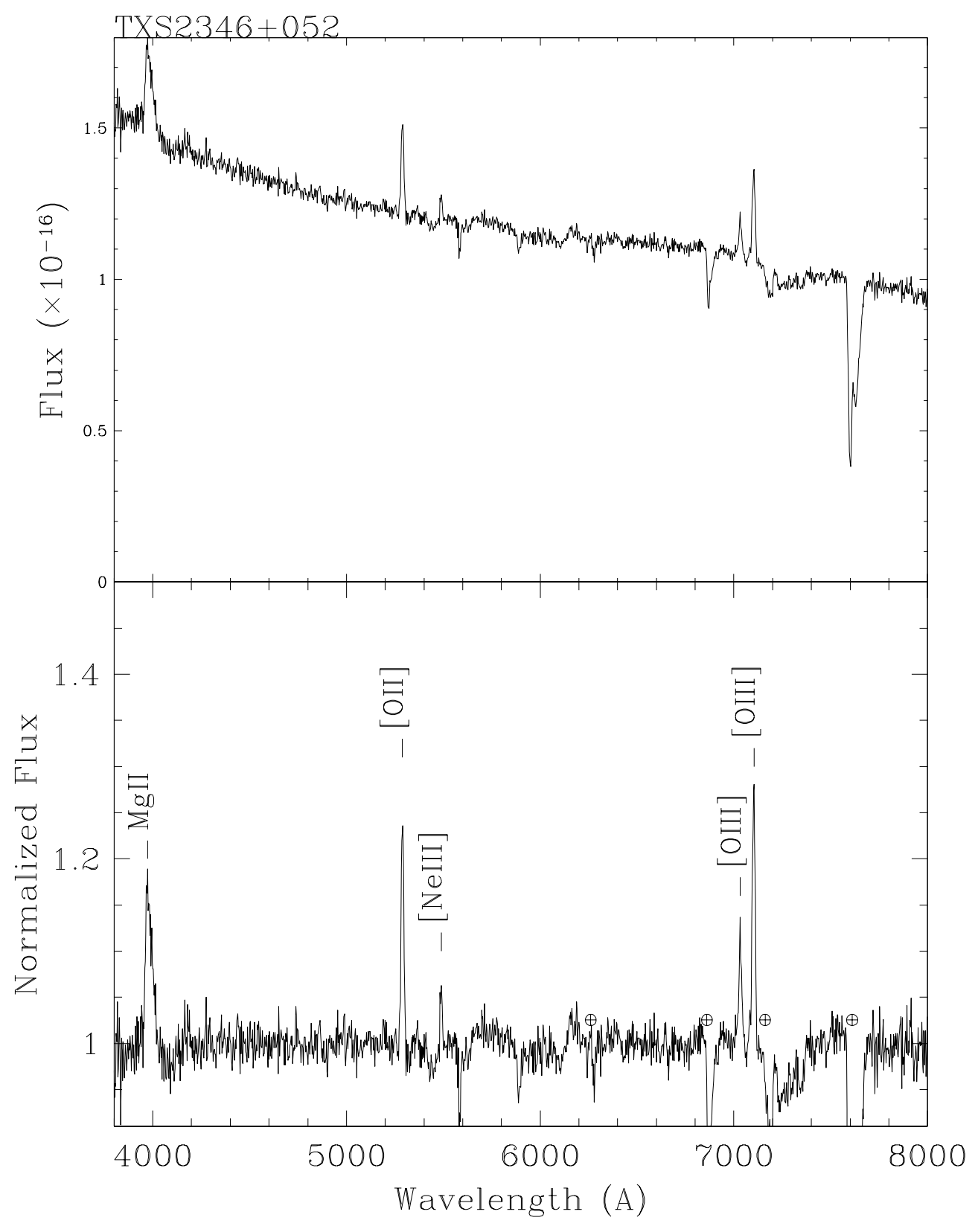

Figure 1:-continued. 


\section{References}

[1] B. Sbarufatti, A. Treves, R. Falomo, J. Heidt, J. Kotilainen, R. Scarpa, ESO Very Large Telescope optical spectroscopy of BL Lacertae. I. New redshifts. AJ 2005 (129) 559

[2] B. Sbarufatti, A. Treves, R. Falomo, J. Heidt, J. Kotilainen, R. Scarpa, ESO Very Large Telescope optical spectroscopy of BL Lacertae. II. New redshifts, featureless objects, and classification assessments. AJ 2006 (132) 1

[3] B. Sbarufatti, S. Ciprini, J. Kotilainen, R. Decarli, A. Treves, A. Veronesi, R. Falomo, ESO VLT optical spectroscopy of BL Lacertae objects. III. An extension of the sample. 2008 AJ in press. 